\title{
Candida Bloodstream Infections in Italy: Changing Epidemiology during 16 Years of Surveillance
}

\author{
Giuseppina Caggiano, Caterina Coretti, Nicola Bartolomeo, Grazia Lovero, \\ Osvalda De Giglio, and Maria Teresa Montagna \\ Hygiene Section, Department of Biomedical Science and Human Oncology, University of Bari, Piazza G. Cesare 11, 70124 Bari, Italy \\ Correspondence should be addressed to Giuseppina Caggiano; giuseppina.caggiano@uniba.it
}

Received 4 December 2014; Revised 18 April 2015; Accepted 20 April 2015

Academic Editor: Handan Wand

Copyright (c) 2015 Giuseppina Caggiano et al. This is an open access article distributed under the Creative Commons Attribution License, which permits unrestricted use, distribution, and reproduction in any medium, provided the original work is properly cited.

\begin{abstract}
Although considerable progress has been made in the management of patients with invasive fungal infections, Candida bloodstream infections are still widespread in hospital settings. Incidence rates vary geographically, often because of different patient populations. The aim of the present study was to describe the epidemiology of candidemia, to analyze the trend of species distribution, and to measure the in vitro susceptibility to antifungal drugs in a university Italian hospital from 1998 to 2013 . The antifungal susceptibility for all Candida isolates was evaluated by broth microdilution assay (CLSI M27-A3 document). Of 394 episodes of candidemia, the average incidence was 3.06/10 000 admissions. C. albicans and non-albicans Candida species caused $44.2 \%$ and $55.8 \%$ of the episodes, respectively. C. parapsilosis (62.2\%) was the most common non-albicans. C. albicans predominated in almost all departments whereas C. parapsilosis was found in adult and paediatric oncohaematology units (34.8\% and $77.6 \%$, resp.). Overall, mortality occurred in 111 (28.2\%) patients. Death occurred most often in intensive care units (47.1\%) and specialist surgeries (43.7\%). Most of the isolates were susceptible to antifungal drugs, but there was an upward trend for azole $(P<0.05)$. In conclusion, this study emphasizes the importance of monitoring local epidemiologic data and the diversity of patient groups affected.
\end{abstract}

\section{Introduction}

For over 20 years, Candida bloodstream infections (BSIs) have been increasing significantly worldwide, representing an important infective complication in hospitalized patients with medical and surgical disorders [1]. Candida spp. are the fourth leading cause of nosocomial BSIs in the USA and the sixth in Europe [2-4]. Though the Candida species distribution varies, there is a well-documented diffusion of Candida albicans across geographical regions [5]. The epidemiology of candidemia generally appears complex and is characterized by a substantial regional and temporal variability. In fact, different incidence rates and new emerging species vary geographically in frequency of isolation, often because of different patient populations or study methodologies [6-8]. Despite advances in medical interventions, the development of novel antifungal drugs, and the infrequent resistance to common antifungal agents, mortality rates are still unacceptably high, ranging from $29 \%$ to $76 \%$, with an attributable mortality of $49 \%$ [1]. This issue is largely due to factors such as the lack of specific clinical findings, the relatively slow and insensitive diagnostic tests that complicate the early recognition of fungal infections, and delays in the administration of appropriate therapy. The aim of this study was to describe the epidemiology of Candida BSIs in a hospital in Southern Italy during 16 years of observation and to analyze the trend in species distribution and in vitro susceptibility to common antifungal drugs.

\section{Patients and Methods}

2.1. Study Design. This observational study was carried out from January 1998 to December 2013 in a university hospital in Apulia, Southern Italy. The hospital is a 1400-bed teaching hospital that attends to cases of high complexity in medicine and surgery, including solid organ and haematopoietic stem cell transplants. All patients with BSI caused by Candida spp., when isolates were available, were included. An episode of 
candidemia was defined as Candida infection involving at least one blood culture.

For each patient, only the first episode of Candida BSI was recorded. Events were considered separate if they occurred at least 30 days apart or were caused by different species. Patients with candidemia were followed prospectively for 30 days after diagnosis.

Neither ethical approval nor patient consensus was considered necessary because we did not use patient accessible data or additional samples other than those obtained during the routine activity of laboratories. All data were collected using standardized case report forms.

2.2. Laboratory Procedures. Blood cultures were made using a lysis-centrifugation system (Isolator, DuPont Co., Wilmington, DE, USA). The samples were cultured on two plates of Sabouraud dextrose agar with $0.05 \%$ chloramphenicol (BioRad, Marnes-la-Coquette, France), incubated at $36^{\circ} \mathrm{C}( \pm 1)$ and $28^{\circ} \mathrm{C}( \pm 1)$, and examined daily for 10 days. The yeast isolates were identified by analysis of biochemical patterns by ID32C kit (bioMèrieux, Marcy l'Étoile, France) and were frozen at $-70^{\circ} \mathrm{C}$ until further investigation.

The antifungal susceptibility tests were performed for all Candida spp. isolates. The following drugs were supplied by the manufacturers as pure standard compounds: anidulafungin (AND), fluconazole (FLC), voriconazole (VRC) (Pfizer Pharmaceuticals, Groton, CT, USA), caspofungin (CSP) (Merck \& Co, Inc., Whitehouse Station, NJ, USA), posaconazole (PSC) (Schering-Plough Corporation, Kenilworth, NJ, USA), micafungin (MCF) (Astellas Pharma, Tokyo, Japan), and amphotericin B (AmB) obtained from Sigma (Sigma-Aldrich, Milan, Italy). Antifungal susceptibility was evaluated by a broth microdilution assay performed according to the Clinical and Laboratory Standards Institute recommendations (CLSI M27-A3 document) [13]. Before the beginning of the study, the isolates were subcultured on antimicrobial agent-free medium to ensure viability and purity. C. parapsilosis ATCC 22019 and C. krusei ATCC 6258 (American Type Culture Collection, Manassas, VA) were used as quality controls and tested in each run of the experiments. All tests were performed in duplicate. MIC (minimum inhibitory concentration) values were determined visually as the lowest concentration of drug that caused significant growth diminution levels $(\geq 50 \%)$. The MIC for $\mathrm{AmB}$ was defined as the lowest drug concentration at which growth was completely inhibited (100\%).

The susceptibility values were interpreted taking into account the new species-specific clinical breakpoints suggested by the CLSI subcommittee for the most common species of Candida $[9,10]$. Interpretive criteria for AmB and PSC have not been established, so based on reported data $[11,12]$ we selected a breakpoint of $>1.0 \mathrm{mcg} / \mathrm{mL}$ to define isolates as $\mathrm{AmB}$ resistant and the species-specific breakpoints for VRC were used for PSC MIC values. The epidemiological cut-off value (ECV) of $\geq 1 \mu \mathrm{g} / \mathrm{mL}$ was used to detect resistance in C. glabrata [9] (Table 1).

2.3. Statistical Analysis. The baseline characteristics of the patients included in the study were expressed by means, medians, and percentages. The incidence rate was calculated as the ratio between the number of new cases reported each year in the wards and the total number of patients hospitalized in the same facility. The association between qualitative variables was assessed by the chi-squared test. Through a univariate logistic model we tested the effect of each variable on the probability of death. Variables found to be significant in the univariate analysis were included in a multivariate logistic model. In all comparisons, a $P<0.05$ was considered statistically significant. Data analysis was performed using the Statistical Package for the Social Sciences (SPSS) software 10 for Mac OS X (SPSS Inc., Chicago, IL, USA).

\section{Results}

During the 16 years of the study, 394 episodes of candidemia were identified, with an average incidence of 3.06/10 000 admissions (range $0.81-6.84$ ). The median age of patients was 49.5 years (interquartile range, 9-67) and more candidemia occurred among males (65.7\%). Most patients had one or more predisposing factors for candidemia: the use of broadspectrum antibiotic treatment (for $>15$ days) was documented in $86.8 \%(342 / 394)$ of the cases, $340 / 394(86.3 \%)$ patients had an intravascular catheter that only in $67 \%$ (228/340) of cases was removed, and $34.3 \%$ (135/394) of patients received total parenteral nutrition. At the time of candidemia diagnosis, $31.2 \%$ of patients (123/394) received antifungal prophylaxis with FLC (mean treatment 14.42 days, range 2-32). Out of 394 Candida BSIs, 123 (31.2\%) were diagnosed in the intensive care unit (ICU); 66 (16.8\%) in the neonatal intensive care unit (NICU); 61 (15.5\%) in general internal medicine; 57 (14.5\%) in general surgery; 49 (12.4\%) in paediatric oncohaematology; $23(5.8 \%)$ in adult oncohaematology; and $15(3.8 \%)$ in specialist surgery (neurosurgery and cardiac surgery).

Antifungal therapy was administered in 75.6\% (298/394) of patients. Of these, liposomal AmB was the most frequently administered drug (182/298; 61.1\%), whereas FLC was employed in $16.8 \%(50 / 298)$ of cases, CSP in $15.4 \%$ (46/298), and MCF in 3.4\% (10/298). Combined therapy was administered in ten patients $(10 / 298 ; 3.4 \%)$, six of whom received liposomal $\mathrm{AmB}$ plus 5-fluorocytosine and four of whom received VRC plus CSP.

During the years of observation, the Candida BSI incidence rate varied, increasing from 2.34 cases per 10000 admissions in the year 1998 to 4.39 cases per 10000 admissions in 2000, whereas in 2006 the incidence decreased significantly to 1.16 cases per 10000 admissions $(P<0.0001)$. After a peak of 6.84 and 6.49 cases per 10000 admissions in the years 2007 and 2008, respectively, the incidence rate decreased consistently and significantly to 2.86 cases per 10000 admissions in the year $2013(P<0.0001)$ (Figure 1$)$.

Considering the global distribution of species, C. albicans and non-albicans species caused 44.2\% (174/394) and 55.8\% (220/394) of the episodes, respectively. A slight variable drift of temporal trend of Candida spp. was observed during the 16 years of study, with a considerable percentage (75\%) increase in non-albicans species in 2004 and 2013 (Figure 2). Among 220 non-albicans Candida included C. parapsilosis (137; 62.2\%), C. glabrata (22; 10\%), C. tropicalis (19;8.6\%), 
TABLE 1: Species-specific breakpoints for antifungal agents for the most common species of Candida isolates.

\begin{tabular}{|c|c|c|c|c|c|}
\hline \multirow{2}{*}{ Species } & \multirow{2}{*}{ Antifungal agent } & \multicolumn{4}{|c|}{$\mathrm{MIC}(\mu \mathrm{g} / \mathrm{mL})$} \\
\hline & & S & SDD & I & $\mathrm{R}$ \\
\hline \multirow{7}{*}{ C. albicans } & Amphotericin B & $<1$ & & & $\geq 1$ \\
\hline & Fluconazole & $\leq 2$ & 4 & & $\geq 8$ \\
\hline & Voriconazole & $\leq 0.12$ & & $0.25-0.5$ & $\geq 1$ \\
\hline & Posaconazole & $\leq 0.12$ & & $0.25-0.5$ & $\geq 1$ \\
\hline & Anidulafungin & $\leq 0.25$ & & 0.5 & $\geq 1$ \\
\hline & Caspofungin & $\leq 0.25$ & & 0.5 & $\geq 1$ \\
\hline & Micafungin & $\leq 0.25$ & & 0.5 & $\geq 1$ \\
\hline \multirow{7}{*}{ C. parapsilosis } & Amphotericin B & $<1$ & & & $\geq 1$ \\
\hline & Fluconazole & $\leq 2$ & 4 & & $\geq 8$ \\
\hline & Voriconazole & $\leq 0.12$ & & $0.25-0.5$ & $\geq 1$ \\
\hline & Posaconazole & $\leq 0.12$ & & $0.25-0.5$ & $\geq 1$ \\
\hline & Anidulafungin & $\leq 2$ & & 4 & $\geq 8$ \\
\hline & Caspofungin & $\leq 2$ & & 4 & $\geq 8$ \\
\hline & Micafungin & $\leq 2$ & & 4 & $\geq 8$ \\
\hline \multirow{7}{*}{ C. glabrata } & Amphotericin B & $<1$ & & & $\geq 1$ \\
\hline & Fluconazole & & $\leq 32$ & & $\geq 64$ \\
\hline & Voriconazole & & & & $\geq 1$ \\
\hline & Posaconazole & & & & $\geq 1$ \\
\hline & Anidulafungin & $\leq 0.12$ & & 0.25 & $\geq 0.5$ \\
\hline & Caspofungin & $\leq 0.12$ & & 0.25 & $\geq 0.5$ \\
\hline & Micafungin & $\leq 0.06$ & & 0.12 & $\geq 0.25$ \\
\hline \multirow{7}{*}{ C. guilliermondii } & Amphotericin B & $<1$ & & & $\geq 1$ \\
\hline & Fluconazole & & & & \\
\hline & Voriconazole & & & & \\
\hline & Posaconazole & & & & \\
\hline & Anidulafungin & $\leq 2$ & & 4 & $\geq 8$ \\
\hline & Caspofungin & $\leq 2$ & & 4 & $\geq 8$ \\
\hline & Micafungin & $\leq 2$ & & 4 & $\geq 8$ \\
\hline \multirow{7}{*}{ C. tropicalis } & Amphotericin B & $<1$ & & & $\geq 1$ \\
\hline & Fluconazole & $\leq 2$ & 4 & & $\geq 8$ \\
\hline & Voriconazole & $\leq 0.12$ & & $0.25-0.5$ & $\geq 1$ \\
\hline & Posaconazole & $\leq 0.12$ & & $0.25-0.5$ & $\geq 1$ \\
\hline & Anidulafungin & $\leq 0.25$ & & 0.5 & $\geq 1$ \\
\hline & Caspofungin & $\leq 0.25$ & & 0.5 & $\geq 1$ \\
\hline & Micafungin & $\leq 0.25$ & & 0.5 & $\geq 1$ \\
\hline \multirow{7}{*}{ C. krusei } & Amphotericin B & $<1$ & & & $\geq 1$ \\
\hline & Fluconazole & - & - & & - \\
\hline & Voriconazole & $\leq 0.5$ & & 1 & $\geq 2$ \\
\hline & Posaconazole & $\leq 0.5$ & & 1 & $\geq 2$ \\
\hline & Anidulafungin & $\leq 0.25$ & & 0.5 & $\geq 1$ \\
\hline & Caspofungin & $\leq 0.25$ & & 0.5 & $\geq 1$ \\
\hline & Micafungin & $\leq 0.25$ & & 0.5 & $\geq 1$ \\
\hline
\end{tabular}

Data compiled from [9-12].

S, susceptible; SDD, susceptible-dose-dependent; I, intermediate; R, resistant. All C. krusei isolates were considered intrinsically resistant to fluconazole [9].

C. guilliermondii (19; 8.6\%), and C. krusei (11; 5\%) whereas other species, C. lusitaniae, C. norvegensis, C. inconspicua, $C$. famata, C. intermedia, C. zeylanoides, and C. pelliculosa, were relatively rare $(12 ; 5.4 \%)$.
The dispersion of $C$. albicans and non-albicans isolates differed according to the patient population. C. albicans predominated in almost all departments, particularly in general surgery (63.2\%), neonatal intensive care unit (54.4\%), 


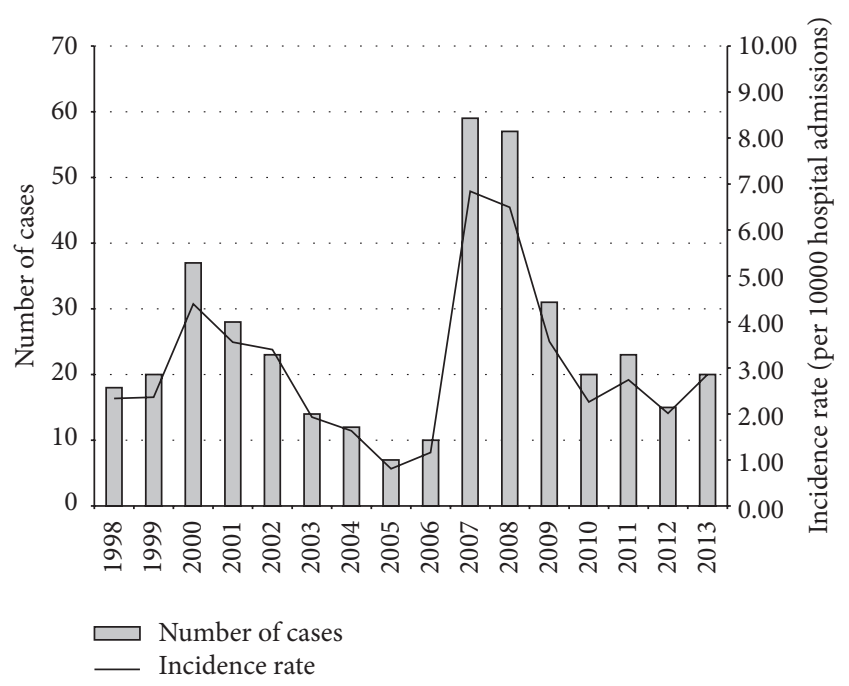

FIgure 1: Patients with Candida BSI and incidence rate observed during a 16-year period.

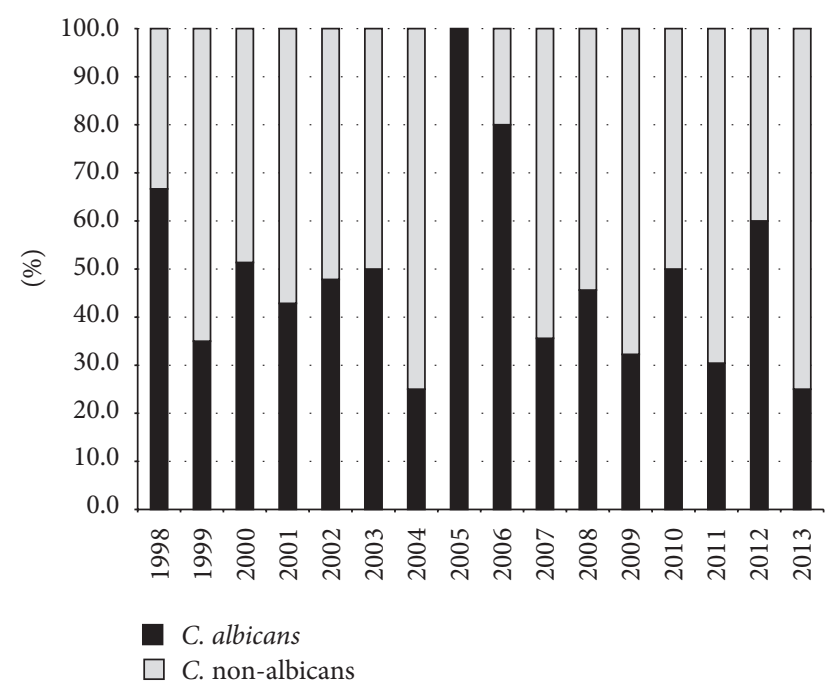

Figure 2: Temporal trend of Candida albicans and non-albicans during a 16-year period.

and general internal medicine (42.2\%), whereas C. parapsilosis was the most frequently isolated species in adult and paediatric oncohaematology units $(34.8 \%$ and $77.6 \%$, resp.) (Figure 3).

C. parapsilosis showed a predilection toward younger patients of 1-17 years of age (39/137, 28.5\%), whereas C. albicans was most frequently isolated in the age groups older than 40 years $(113 / 174 ; 65.9 \%)$. Fourteen of $22(63.6 \%)$ C. glabrata BSI cases were diagnosed in the age range 41-80 years. $C$. krusei was present in adults, in particular in the age groups of 41-80 years, whereas it was absent in children (Table 2).

Out of 123 patients (31.2\%) receiving antifungal prophylaxis at the time of candidemia, $22(17.9 \%)$ developed $C$. albicans BSI and the remaining 101 (82.1\%) developed nonalbicans infection, in particular by C. parapsilosis (61/101;

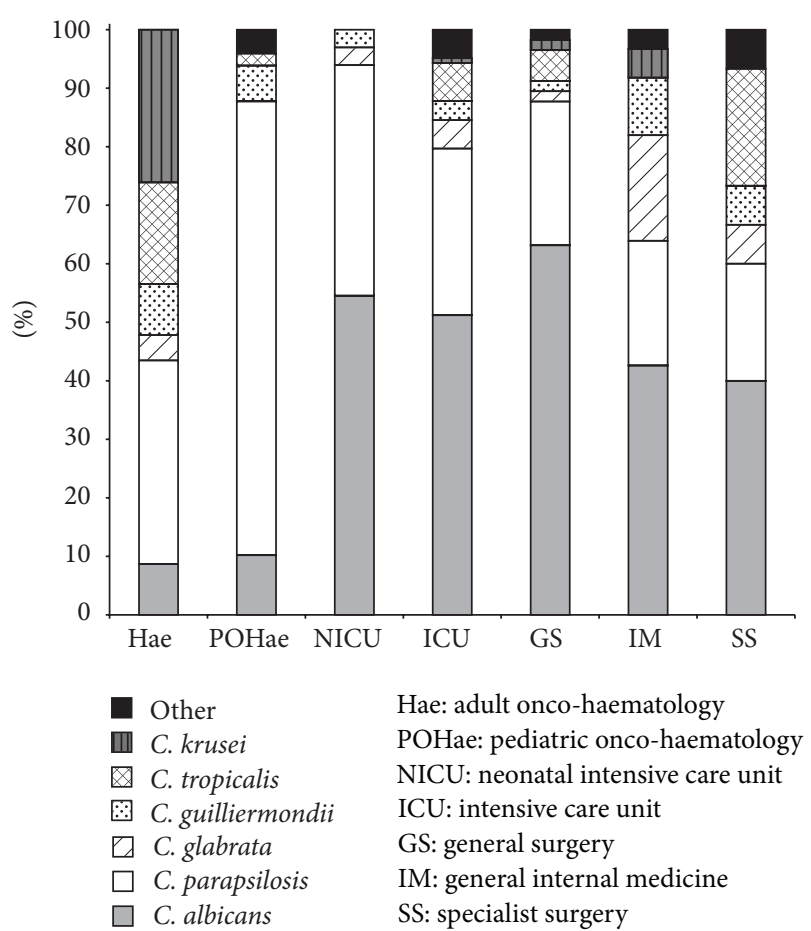

Figure 3: Distribution of Candida species according to hospital departments.

60.4\%), C. glabrata (11/101; 10.9\%), C. tropicalis (9/101; 8.9\%), C. krusei (8/101; 7.9\%), and C. guilliermondii (7/101; 6.9\%). A significant dependence between prophylaxis and isolated Candida species resulted $(P<0.0001)$.

Overall, mortality occurred in $111(28.2 \%)$ candidemic patients within 20 days from the onset of candidemia. Of those, 48 (43.2\%) patients had BSI due to C. albicans and 63 $(56.7 \%)$ to non-albicans species. We observed a significant dependence between death and hospital setting $(P<0.001)$ (Table 3); cases of death were higher in the ICU (58/123; $47.1 \%)$ and in specialist surgery $(7 / 16 ; 43.7 \%)$.

A multivariate logistic model was built with the significant variables from a univariate analysis. The covariate "age," "species," and "department" were significant (age $[P=0.005]$, species $[P=0.012]$, and department $[P=0.001])$. The Hosmer-Lemeshow Goodness-of-Fit of the model indicated that observed data matched expected data (chi-squared = 7.541; $P=0.480$ ). The multivariate analysis indicated that $C$. krusei was associated with a statistically higher risk of death compared with both $C$. albicans $(\mathrm{OR}=6.478 ; \mathrm{CL}=1.450$, 28.935) and C. parapsilosis ( $\mathrm{OR}=7.909 ; \mathrm{CL}=1.731,36.130)$. C. glabrata was also associated with a three times higher risk than $C$. parapsilosis $(\mathrm{OR}=3.226 ; \mathrm{CL}=1.133,9.185)$. In our analysis, age presented a risk of death associated with OR of $1.019(\mathrm{CL}=1.006,1.033)$ for an increase of 1 year of age.

Table 4 shows the results of in vitro susceptibility testing of the most commonly isolated species of Candida $(n=382)$. Most of the isolates were susceptible to the antifungal drugs tested. All azoles demonstrated good activity. In particular, only 5\% (18/363) and 2.9\% (10/341) of isolates were resistant to FLC and VRC, respectively. Resistance for both drugs was 
TABLE 2: Distribution of Candida spp. isolates for different age groups.

\begin{tabular}{|c|c|c|c|c|c|c|c|c|c|c|c|c|c|c|}
\hline \multirow{3}{*}{ Candida species } & \multicolumn{14}{|c|}{ Patients age groups } \\
\hline & \multicolumn{2}{|c|}{$<1$} & \multicolumn{2}{|c|}{$1-17$} & \multicolumn{2}{|c|}{$18-40$} & \multicolumn{2}{|c|}{$41-60$} & \multicolumn{2}{|c|}{$61-80$} & \multicolumn{2}{|c|}{$>80$} & \multicolumn{2}{|c|}{ Total } \\
\hline & $n$ & $\%$ & $n$ & $\%$ & $n$ & $\%$ & $n$ & $\%$ & $n$ & $\%$ & $n$ & $\%$ & $n$ & $\%$ \\
\hline C. albicans & 33 & 19.0 & 12 & 6.9 & 16 & 9.2 & 49 & 28.2 & 53 & 30.5 & 11 & 6.3 & 174 & 100 \\
\hline C. parapsilosis & 25 & 18.2 & 39 & 28.5 & 13 & 9.5 & 27 & 19.7 & 25 & 18.2 & 8 & 5.8 & 137 & 100 \\
\hline C. glabrata & 2 & 9.1 & 0 & 0.0 & 3 & 13.6 & 5 & 22.7 & 9 & 40.9 & 3 & 13.6 & 22 & 100 \\
\hline C. guilliermondii & 2 & 10.5 & 5 & 26.3 & 1 & 5.3 & 5 & 26.3 & 3 & 15.8 & 3 & 15.8 & 19 & 100 \\
\hline C. tropicalis & 0 & 0.0 & 2 & 10.5 & 1 & 5.3 & 7 & 36.8 & 9 & 47.4 & 0 & 0.0 & 19 & 100 \\
\hline C. krusei & 0 & 0.0 & 1 & 9.1 & 1 & 9.1 & 5 & 45.5 & 4 & 36.4 & 0 & 0.0 & 11 & 100 \\
\hline Other Candida spp.* & 0 & 0.0 & 2 & 16.7 & 4 & 33.3 & 2 & 16.7 & 2 & 16.7 & 2 & 16.7 & 12 & 100 \\
\hline Total & 62 & 15.7 & 61 & 15.5 & 39 & 9.9 & 100 & 25.4 & 105 & 26.6 & 27 & 6.9 & 394 & 100 \\
\hline
\end{tabular}

${ }^{*}$ Candida spp.: C. lusitaniae, C. norvegensis, C. inconspicua, C. famata, C. intermedia, C. zeylanoides, and C. pelliculosa.

TABLE 3: Risk analysis of death with a univariate logistic mode.

\begin{tabular}{|c|c|c|c|}
\hline & $\begin{array}{c}\text { Death } \\
N=111\end{array}$ & $\begin{array}{c}\text { Living } \\
N=283\end{array}$ & $p$ (Wald test) \\
\hline Age, years (median, I and III quartile) & $58.0(44.0-74.0)$ & $42.0(8.0-64.0)$ & $<0.0001^{*}$ \\
\hline \multicolumn{4}{|l|}{ Gender, $n(\%)$} \\
\hline Male & $72(64.9)$ & $187(66.1)$ & \multirow{2}{*}{0.819} \\
\hline Female & $39(35.1)$ & $96(33.9)$ & \\
\hline \multicolumn{4}{|l|}{ Catheter, $n .(\%)$} \\
\hline Yes & $99(89.2)$ & $241(85.2)$ & \multirow{2}{*}{0.128} \\
\hline No & $12(10.8)$ & $42(14.8)$ & \\
\hline \multicolumn{4}{|l|}{ Antibiotic therapy, $n$. (\%) } \\
\hline Yes & $97(87.4)$ & $245(86.6)$ & \multirow{2}{*}{0.830} \\
\hline No & $14(12.6)$ & $38(13.4)$ & \\
\hline \multicolumn{4}{|l|}{ Antifungal prophylaxis, $n$. (\%) } \\
\hline Yes & $36(32.4)$ & $87(30.7)$ & \multirow{2}{*}{0.745} \\
\hline No & $75(67.6)$ & $196(69.3)$ & \\
\hline \multicolumn{4}{|l|}{ Candida species, $n .(\%)$} \\
\hline C. albicans & $48(43.2)$ & $126(44.5)$ & \multirow{7}{*}{0.001} \\
\hline C. parapsilosis & $24(21.6)$ & $113(9.9)$ & \\
\hline C. glabrata & $11(9.9)$ & $11(3.9)$ & \\
\hline C. guilliermondii & $7(6.3)$ & $12(4.2)$ & \\
\hline C. tropicalis & $8(7.2)$ & $11(3.9)$ & \\
\hline C. krusei & $6(5.4)$ & $5(1.8)$ & \\
\hline Other & $7(6.3)$ & $5(1.8)$ & \\
\hline \multicolumn{4}{|l|}{ Department, $n$. (\%) } \\
\hline Adult oncohaematology & $5(4.5)$ & $18(6.4)$ & \multirow{7}{*}{$<0.001^{* *}$} \\
\hline Pediatric oncohaematology & - & $49(17.3)$ & \\
\hline NICU & $12(10.8)$ & $54(19.1)$ & \\
\hline $\mathrm{ICU}$ & $58(52.2)$ & $65(23)$ & \\
\hline General surgery & $12(10.8)$ & $45(15.9)$ & \\
\hline General internal medicine & $17(15.3)$ & $43(15.2)$ & \\
\hline Specialist surgery & $7(6.3)$ & $8(2.8)$ & \\
\hline
\end{tabular}

NICU: neonatal intensive care unit.

ICU: intensive care unit.

* Wilcoxon test.

${ }^{* *}$ The pediatric haematology patients were excluded from analysis because none of the candidemic patients died. 
TABLE 4: In vitro susceptibility to the antifungal agents against 394 Candida isolates.

\begin{tabular}{|c|c|c|c|c|}
\hline \multirow{2}{*}{$\begin{array}{l}\text { Species } \\
\text { (number of isolates) }\end{array}$} & \multirow{2}{*}{ Antifungal agent } & \multicolumn{3}{|c|}{$\mathrm{MIC}(\mu \mathrm{g} / \mathrm{mL})$} \\
\hline & & Range & $50 \%$ & $90 \%$ \\
\hline \multirow{7}{*}{ C. albicans (174) } & Amphotericin B & $0.06-1$ & 0.50 & 1 \\
\hline & Fluconazole & $0.06-2$ & 0.25 & 1 \\
\hline & Voriconazole & $\leq 0.008-0.5$ & 0.008 & 0.25 \\
\hline & Posaconazole & $\leq 0.008-0.5$ & 0.03 & 0.25 \\
\hline & Anidulafungin & $\leq 0.008-1$ & 0.06 & 0.25 \\
\hline & Caspofungin & $\leq 0.008-1$ & 0.25 & 0.5 \\
\hline & Micafungin & $\leq 0.008-0.5$ & 0.03 & 0.06 \\
\hline \multirow{7}{*}{ C. parapsilosis (137) } & Amphotericin B & $0.06-1$ & 0.25 & 0.5 \\
\hline & Fluconazole & $0.06-8$ & 0.5 & 1 \\
\hline & Voriconazole & $\leq 0.008-1$ & 0.015 & 0.06 \\
\hline & Posaconazole & $\leq 0.008-2$ & 0.125 & 0.25 \\
\hline & Anidulafungin & $\leq 0.008-32$ & 1 & 2 \\
\hline & Caspofungin & $0.125-8$ & 2 & 2 \\
\hline & Micafungin & $\leq 0.008-32$ & 1 & 2 \\
\hline \multirow{7}{*}{ C. glabrata (22) } & Amphotericin B & $0.25-1$ & 0.5 & 1 \\
\hline & Fluconazole & $2-32$ & 8 & 16 \\
\hline & Voriconazole & $0.03-2$ & 0.25 & 1 \\
\hline & Posaconazole & $0.25-4$ & 0.25 & 4 \\
\hline & Anidulafungin & $\leq 0.008-1$ & 0.25 & 0.25 \\
\hline & Caspofungin & $\leq 0.008-4$ & 0.06 & 0.5 \\
\hline & Micafungin & $\leq 0.008-1$ & 0.06 & 0.5 \\
\hline \multirow{7}{*}{ C. guilliermondii (19) } & Amphotericin B & $0.125-1$ & 0.25 & 1 \\
\hline & Fluconazole & $0.5-4$ & 4 & 4 \\
\hline & Voriconazole & $\leq 0.008-1$ & 0.06 & 0.5 \\
\hline & Posaconazole & $0.125-1$ & 0.5 & 1 \\
\hline & Anidulafungin & $1-4$ & 1 & 2 \\
\hline & Caspofungin & $1-4$ & 2 & 4 \\
\hline & Micafungin & $0.5-2$ & 1 & 2 \\
\hline \multirow{7}{*}{ C. tropicalis (19) } & Amphotericin B & $0.06-1$ & 0.5 & 1 \\
\hline & Fluconazole & $0.125-64$ & 0.5 & 64 \\
\hline & Voriconazole & $0.008-16$ & 0.06 & 8 \\
\hline & Posaconazole & $0.015-2$ & 0.125 & 0.5 \\
\hline & Anidulafungin & $0.03-0.25$ & 0.25 & 0.25 \\
\hline & Caspofungin & $\leq 0.008-2$ & 0.06 & 1 \\
\hline & Micafungin & $0.0015-1$ & 0.25 & 0.5 \\
\hline \multirow{7}{*}{ C. krusei (11) } & Amphotericin B & $0.5-1$ & 1 & 1 \\
\hline & Fluconazole & $8-64$ & 32 & 32 \\
\hline & Voriconazole & $0.125-8$ & 0.25 & 1 \\
\hline & Posaconazole & $0.25-2$ & 0.5 & 2 \\
\hline & Anidulafungin & $\leq 0.008-0.5$ & 0.25 & 0.5 \\
\hline & Caspofungin & $0.06-2$ & 0.25 & 1 \\
\hline & Micafungin & $0.06-0.5$ & 0.25 & 0.5 \\
\hline
\end{tabular}


expressed mainly by C. krusei and C. tropicalis. PSC was inactive in $1.8 \%(6 / 341)$ of isolates. Similarly, $4.7 \%(18 / 382)$, $2.1 \%(8 / 382)$, and $1.3 \%(5 / 382)$ of isolates were resistant to CSP, AND, and MCF, respectively. Eight out of 174 (4.6\%) C. albicans were resistant to CSP; $36.4 \%$ (4/11) of C. krusei and $21.1 \%(4 / 19)$ of $C$. tropicalis were resistant to CSP. Resistance to AND was mainly recognized in C. parapsilosis. All isolates were susceptible toAmB. When the annual geometric mean MIC values for the individual antifungals were plotted, only three drugs demonstrated an upward trend: FLC $\left(R^{2}=0.27\right.$; $\exp b$ (0.38); $P=0.04)$, VCZ $\left[R^{2}=0.443\right.$; $\exp b(0.031)$; $P=0.005)$, and PCZ $\left(R^{2}=0.335 ; \exp b(0.023) ; P=0.355\right]$.

\section{Discussion}

Although considerable progress has been made in the management of patients with invasive fungal infections in the recent years, Candida BSIs are still widespread in hospital settings and closely associated with extended hospitalization and high healthcare costs. In the present study we evaluated the case trend of Candida BSIs during 16 years and although it was observed that the incidence per year was quite variable (range 0.81 to $6.84 / 10000$ admissions), the mean rate was 3.06/10 000 admissions and was in accordance with those reported from other centres. In fact, in Denmark, Arendrup et al. [14] observed an incidence of 4.1/10 000 admissions, in Israel Rennert et al. [15] reported 5/10 000 admissions, and in China Li et al. [16] reported 5.3/10 000 admissions; on the other side, some studies report higher incidence rates, as in Portugal, with 8.8/10 000 admissions [17], or in Brazil with $18.7 / 10000$ admissions [18]. This variability of fungal infection incidence rates among various geographical areas may reflect differences in healthcare practices among different countries in the populations studied, as well as the methodology adopted by different authors, or the systems for regular surveillance. In fact, in the present study we observed a maximum peak of incidence in the years 2007-2008 due to a rigorous active surveillance of invasive fungal infections that resulted in greater recruitment of cases [19-21].

Over the past 20 years, a shift toward non-albicans Candida species has been reported $[1,29]$, but the precise pattern of causative species varies across countries; hence epidemiological information available for one centre may not be applicable to others. Overall, in our study more than $50 \%$ of candidemia cases were due to non-albicans Candida. We observed a variable drift through 16 years and, in 2013, 75\% of the cases were caused by non-albicans Candida species that were predominant in adult and paediatric patients with haematological malignancies $(65 / 220 ; 29.5 \%)$.

According to other European reports [22-24], we found that C. albicans was the most frequent fungal species followed by C. parapsilosis, responsible for $62.3 \%$ of non-albicans episodes, whereas others report C. glabrata as the most common non-albicans species $[1,25]$. The high profusion of C. parapsilosis could be explained by factors common in hospital settings, such as gastrointestinal colonization, or the affinity for intravascular devices and prosthetic materials. Additionally, C. parapsilosis has been known to colonize the hands of healthcare workers and is often responsible for nosocomial clusters, so its presence in hospital settings may be related to insufficient implementation of infection control measures. In our hospital during 1999, C. parapsilosis caused a BSI outbreak which affected children with haematological malignances, in which all C. parapsilosis isolates were genotypically identical between the strains isolated from the young patients and those isolated from the hands of one of the healthcare workers [26]. Although C. parapsilosis is less virulent than other species, its widespread dispersion and tendency to form biofilm on devices can interfere with antifungal therapy [27].

As in other studies $[22,28,29]$, we observed a correlation of Candida species with patient age. BSIs due to C. parapsilosis showed a predilection toward younger patients, whereas $C$. glabrata and C. tropicalis were recovered from adult patients (>60 years old). The multivariate analysis showed that the species, independent of age and department, predicted the outcome of patients with candidemia. Certainly, the severity of underlying medical conditions also influences the mortality rate in these patients. Although resistance to antifungal agents is not common, the emergence of various non-albicans Candida species indicates the potential for development of antifungal resistance. For example, resistance to FLC is more common in non-albicans Candida, in part due to nonalbicans species that are inherently resistant to antifungal, such as $C$. krusei $[28,30]$. However, the uncontrolled use of echinocandin could promote the emergence of higher MIC values and promote the diffusion of Candida species such as C. parapsilosis or C. guilliermondii against which echinocandins have in vitro relatively less activity. In our study, the susceptibility rate to this drug class was very high during all periods of surveillance. Additionally, despite considerable use of FLC during these 16 years, the susceptibility rate to this agent continued to be very high, whereas the isolation of species historically less sensitive, such as $C$. glabrata and C. krusei, was low amounting to 22/394 and 11/394, respectively. This fact suggests that, apart from the use of FLC, other aspects may be important factors in the wide geographic variability of the species distribution, including demographic characteristics and use of antibiotics. However, it is important to note that the annual geometric mean MIC values of FLC demonstrated an upward trend during the 16 years, and the same tendency was observed for VRC and PSC. These data allow us to propose the possibility of crossreaction among the azoles, considering that VRC and PSC have also been more recently introduced into clinical practice than FLC, underlying the need to monitor susceptibility trends. However, it is also important to consider that antifungal susceptibility testing has the limitation of correlating imperfectly with clinical outcomes. In fact, drug susceptibility does not guarantee clinical success because of the status of the host immune system and the persistence of infecting foci concurrent with illnesses influencing the outcome. Nevertheless, some isolates do not necessarily follow this general pattern. For this reason, knowing the susceptibility in vitro and monitoring the trend of MICs might facilitate the choice of appropriate therapy and provide guidance in the management of fungal infections. Additionally, the correct identification of fungal isolates is important in assessing 
epidemiology and in identifying geographic trends of the resistance profiles of most common Candida species.

\section{Conclusion}

This study emphasizes the importance of monitoring local epidemiologic data, considering the diversity of affected patient groups, knowing the antifungal susceptibility trend, and recognizing the important potential of such information to impact empiric antifungal therapy.

\section{Conflict of Interests}

The authors declare that there is no conflict of interests regarding the publication of this paper.

\section{Acknowledgment}

This study was supported by an unrestricted grant from Pfizer Italia.

\section{References}

[1] D. Diekema, S. Arbefeville, L. Boyken, J. Kroeger, and M. Pfaller, "The changing epidemiology of healthcare-associated candidemia over three decades," Diagnostic Microbiology and Infectious Disease, vol. 73, no. 1, pp. 45-48, 2012.

[2] R. Ben-Ami, M. Weinberger, R. Orni-Wasserlauff et al., "Time to blood culture positivity as a marker for catheter-related candidemia," Journal of Clinical Microbiology, vol. 46, no. 7, pp. 2222-2226, 2008.

[3] H. Wisplinghoff, T. Bischoff, and S. M. Tallent, "Nosocomial bloodstream infections in US hospitals: analysis of 24,179 cases from a prospective nationwide surveillance study," Clinical Infectious Diseases, vol. 9, pp. 309-317, 2004.

[4] J. Ericsson, E. Chryssanthou, L. Klingspor et al., "Candidaemia in Sweden: a nationwide prospective observational survey," Clinical Microbiology and Infection, vol. 19, no. 4, pp. E218-E221, 2013.

[5] M. A. Pfaller and D. J. Diekema, "Epidemiology of invasive candidiasis: a persistent public health problem," Clinical Microbiology Reviews, vol. 20, no. 1, pp. 133-163, 2007.

[6] J. Pemán, E. Cantón, G. Quindós et al., "Epidemiology, species distribution and in vitro antifungal susceptibility of fungaemia in a Spanish multicentre prospective survey," Journal of Antimicrobial Chemotherapy, vol. 67, no. 5, pp. 1181-1187, 2012.

[7] J. Kriengkauykiat, J. I. Ito, and S. S. Dadwal, "Epidemiology and treatment approaches in management of invasive fungal infections," Clinical Epidemiology, vol. 3, no. 1, pp. 175-191, 2011.

[8] F. G. de Rosa, E. M. Trecarichi, C. Montrucchio et al., "Mortality in patients with early- or late-onset candidaemia," Journal of Antimicrobial Chemotherapy, vol. 68, no. 4, pp. 927-935, 2013.

[9] M. A. Pfaller, D. Andes, M. C. Arendrup et al., "Clinical breakpoints for voriconazole and Candida spp. revisited: review of microbiologic, molecular, pharmacodynamic, and clinical data as they pertain to the development of species-specific interpretive criteria," Diagnostic Microbiology and Infectious Disease, vol. 70, no. 3, pp. 330-343, 2011.

[10] M. A. Pfaller and D. J. Diekema, "Microdilution methods, 2010 to 2012 and laboratory standards institute broth testing of Candida spp. by use of clinical progress in antifungal susceptibility," Journal of Clinical Microbiology, vol. 50, no. 9, pp. 2846-2856, 2012.

[11] M. A. Pfaller, D. J. Diekema, R. N. Jones et al., "International surveillance of bloodstream infections due to Candida species: frequency of occurrence and in vitro susceptibilities to fluconazole, ravuconazole, voriconazole of isolates collected from 1997 through 1999 in the SENTRY antimicrobial surveillance program," Journal of Clinical Microbiology, vol. 39, no. 9, pp. 3254-3259, 2001.

[12] D. J. Diekema, S. A. Messer, L. B. Boyken et al., "In vitro activity of seven systemically active antifungal agents against a large global collection of rare Candida species as determined by CLSI broth microdilution methods," Journal of Clinical Microbiology, vol. 47, no. 10, pp. 3170-3177, 2009.

[13] Clinical and Laboratory Standards Institute, "Reference method for broth dilution antifungal susceptibility testing of yeaststhird informational supplement," Tech. Rep. M27-A3, CLSI, Wayne, Pa, USA, 2008.

[14] M. C. Arendrup, B. Bruun, J. J. Christensen et al., "National surveillance of fungemia in Denmark (2004 to 2009)," Journal of Clinical Microbiology, vol. 49, no. 1, pp. 325-334, 2011.

[15] G. Rennert, H. S. Rennert, S. Pitlik, R. Finkelstein, and R. KitzesCohen, "Epidemiology of candidemia-a nationwide survey in Israel," Infection, vol. 28, no. 1, pp. 26-29, 2000.

[16] D. Li, W. Zhang, S. Zheng, Z. Ma, P. Zhang, and Z. Liu, "Surveillance study of candidemia in cancer patients in North China," Medical Mycology, vol. 51, no. 4, pp. 378-384, 2013.

[17] I. Faria-Ramos, J. Neves-Maia, E. Ricardo et al., "Species distribution and in vitro antifungal susceptibility profiles of yeast isolates from invasive infections during a Portuguese multicenter survey," European Journal of Clinical Microbiology \& Infectious Diseases, vol. 33, no. 12, pp. 2241-2247, 2014.

[18] A. L. Motta, G. M. D. de Almeida, J. N. de Almeida Jr., M. N. Burattini, and F. Rossi, "Candidemia epidemiology and susceptibility profile in the largest Brazilian teaching hospital complex," Brazilian Journal of Infectious Diseases, vol. 14, no. 5, pp. 441-448, 2010.

[19] M. T. Montagna, G. Lovero, O. De Giglio et al., "Invasive fungal infections in Neonatal Intensive Care Units of Southern Italy: a multicentre regional active surveillance (Aurora Project)," Journal of Preventive Medicine and Hygiene, vol. 51, no. 3, pp. 125-130, 2010.

[20] M. T. Montagna, O. de Giglio, C. Napoli et al., "Invasive fungal infections in patients with hematologic malignancies (Aurora Project): lights and shadows during 18-months surveillance," International Journal of Molecular Sciences, vol. 13, no. 1, pp. 774-787, 2012.

[21] M. T. Montagna, G. Caggiano, G. Lovero et al., "Epidemiology of invasive fungal infections in the intensive care unit: results of a multicenter Italian survey (AURORA Project)," Infection, vol. 41, no. 3, pp. 645-653, 2013.

[22] H. Wisplinghoff, J. Ebbers, L. Geurtz et al., "Nosocomial bloodstream infections due to Candida spp. in the USA: species distribution, clinical features and antifungal susceptibilities," International Journal of Antimicrobial Agents, vol. 43, no. 1, pp. 78-81, 2014.

[23] A. Spiliopoulou, S. Vamvakopoulou, C. Bartzavali, G. Dimitracopoulos, E. D. Anastassiou, and M. Christofidou, "Eleven-year retrospective survey of candidaemia in a university hospital in southwestern Greece," Clinical Microbiology and Infection, vol. 16, no. 9, pp. 1378-1381, 2010. 
[24] J. Guinea, Ó. Zaragoza, P. Escribano et al., "Molecular identification and antifungal susceptibility of yeast isolates causing fungemia collected in a population-based study in Spain in 2010 and 2011," Antimicrobial Agents and Chemotherapy, vol. 58, no. 3, pp. 1529-1537, 2014.

[25] E. Matsumoto, L. Boyken, S. Tendolkar et al., "Candidemia surveillance in Iowa: emergence of echinocandin resistance," Diagnostic Microbiology and Infectious Disease, vol. 79, no. 2, pp. 205-208, 2014.

[26] F. Barchiesi, G. Caggiano, L. F. Di Francesco, M. T. Montagna, S. Barbuti, and G. Scalise, "Outbreak of fungemia due to Candida parapsilosis in a pediatric oncology unit," Diagnostic Microbiology \& Infectious Disease, vol. 49, no. 4, pp. 269-271, 2004.

[27] J. C. O. Sardi, L. Scorzoni, T. Bernardi, A. M. Fusco-Almeida, and M. J. S. Mendes Giannini, "Candida species: current epidemiology, pathogenicity, biofilm formation, natural antifungal products and new therapeutic options," Journal of Medical Microbiology, vol. 62, supplement 1, pp. 10-24, 2013.

[28] M. A. Pfaller, M. Castanheira, S. A. Messer, G. J. Moet, and R. N. Jones, "Variation in Candida spp. distribution and antifungal resistance rates among bloodstream infection isolates by patient age: report from the SENTRY Antimicrobial Surveillance Program (2008-2009)," Diagnostic Microbiology and Infectious Disease, vol. 68, no. 3, pp. 278-283, 2010.

[29] M. A. Pfaller, D. R. Andes, D. J. Diekema et al., "Epidemiology and outcomes of invasive candidiasis due to non-albicans species of Candida in 2,496 patients: data from the Prospective Antifungal Therapy (PATH) registry 2004-2008," PLoS ONE, vol. 9, no. 7, Article ID e101510, 2014.

[30] B. D. Alexander, M. D. Johnson, C. D. Pfeiffer et al., "Increasing echinocandin resistance in Candida glabrata: clinical failure correlates with presence of FKS mutations and elevated minimum inhibitory concentrations," Clinical Infectious Diseases, vol. 56, no. 12, pp. 1724-1732, 2013. 


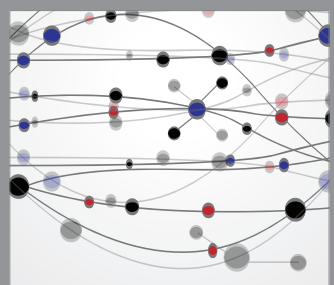

The Scientific World Journal
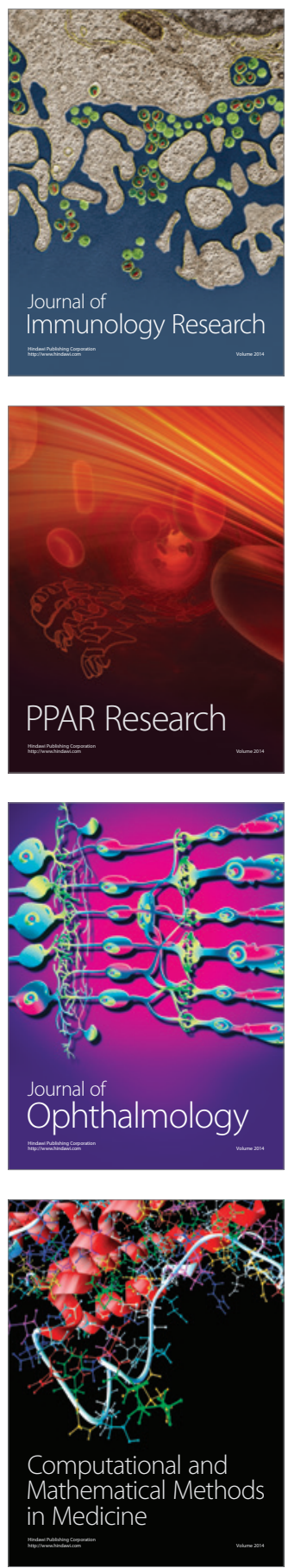

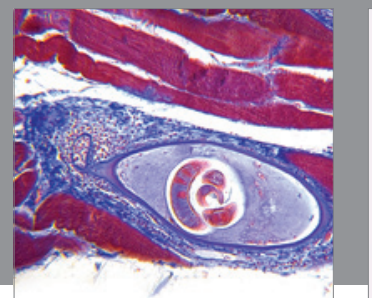

Gastroenterology

Research and Practice
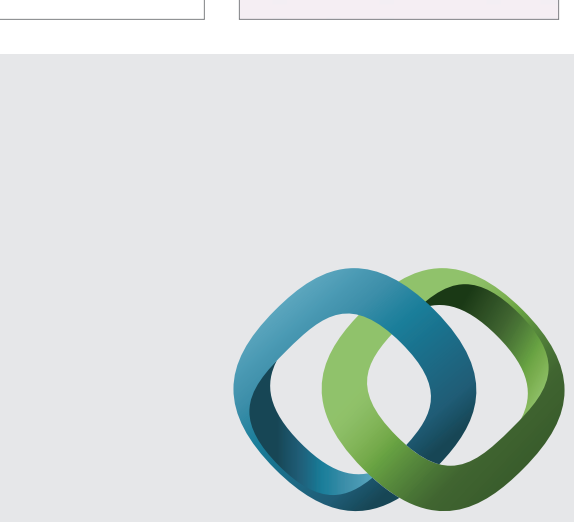

\section{Hindawi}

Submit your manuscripts at

http://www.hindawi.com
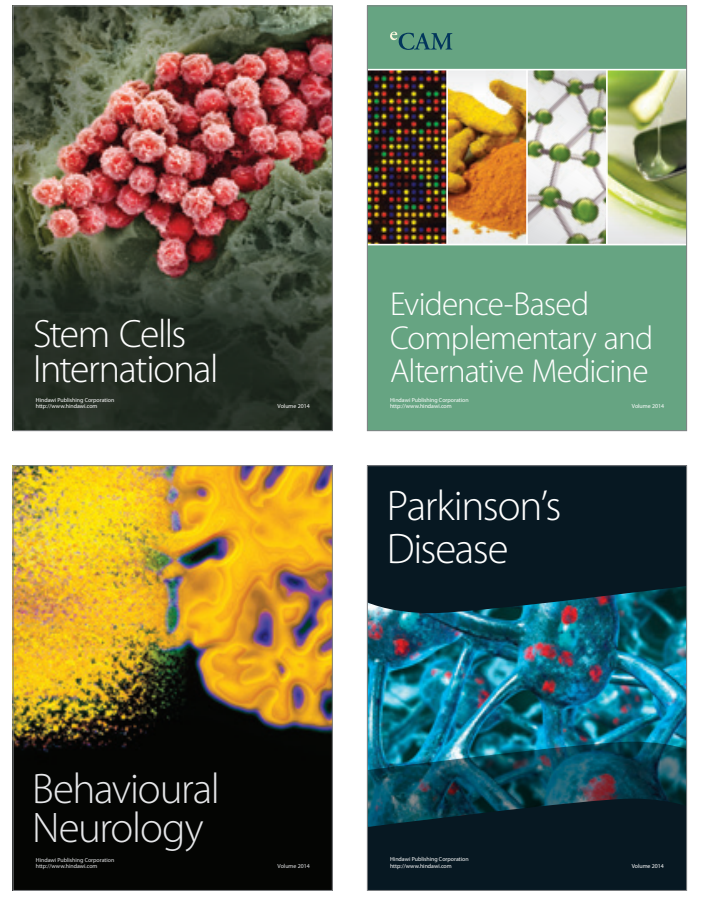
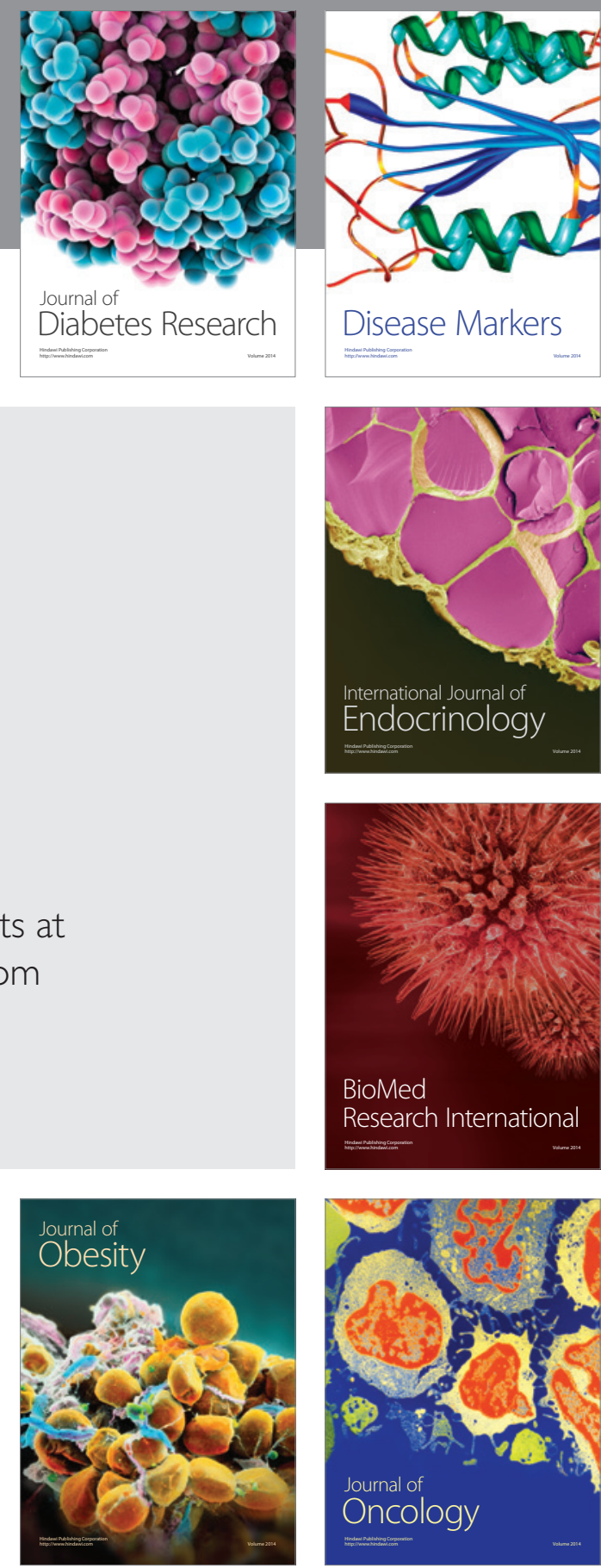

Disease Markers
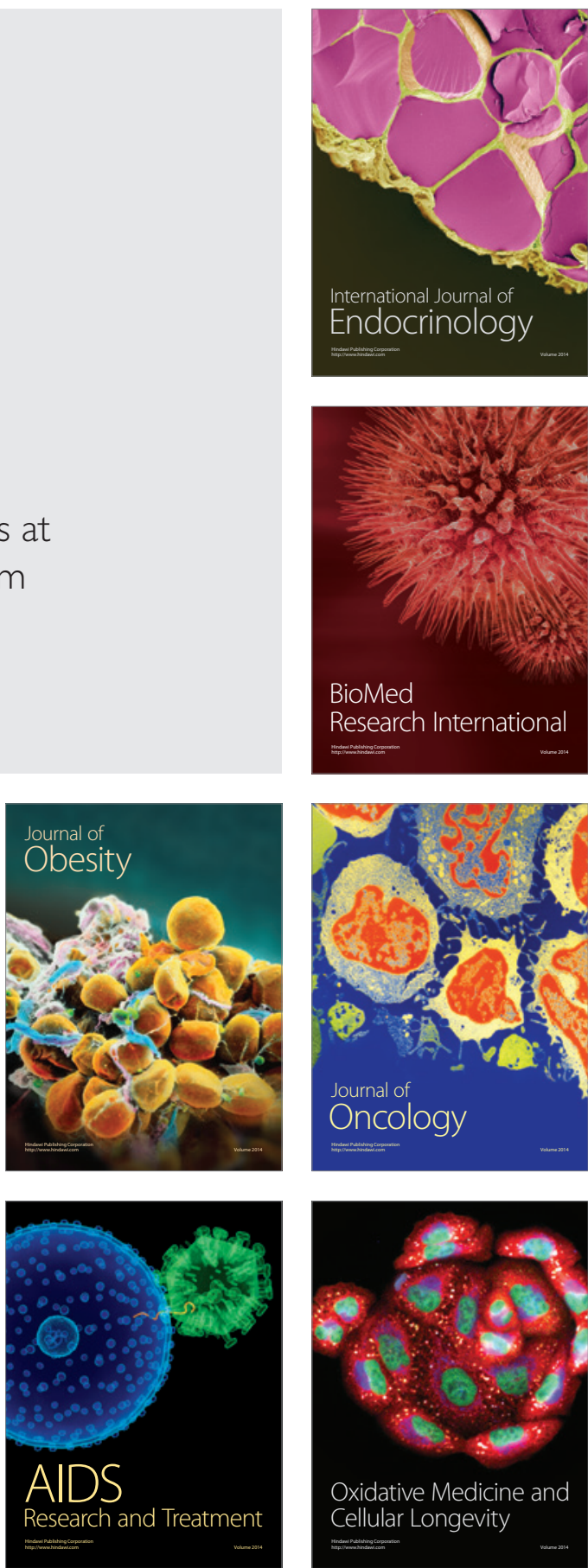